
\title{
28 Research Square \\ The Course of Primary Progressive Aphasia Diagnosis : A Cross-Sectional Study.
}

\section{Aurélie Mouton}

CHU Nice: Centre Hospitalier Universitaire de Nice

\section{Alexandra PLONKA}

University of Cote d'Azur: Universite Cote d'Azur

\section{Roxane FABRE}

CHU Nice: Centre Hospitalier Universitaire de Nice

\section{Maï Tran}

Université de Lille I: Universite de Lille Faculte des Sciences et Technologies

\section{Philippe ROBERT}

Université Côte d'Azur: Universite Cote d'Azur

Joel MACOIR

Laval University: Universite Laval

\section{Valeria MANERA}

Université Côte d'Azur: Universite Cote d'Azur

Auriane GROS ( $\sim$ auriane.gros@univ-cotedazur.fr )

University Côte d'Azur

\section{Research}

Keywords: Primary Progressive Aphasia, Alzheimer's disease, Diagnosis

Posted Date: April 22nd, 2021

DOl: https://doi.org/10.21203/rs.3.rs-440319/v1

License: (c) (1) This work is licensed under a Creative Commons Attribution 4.0 International License.

Read Full License 


\section{Abstract}

\section{Background}

The Primary Progressive Aphasia (PPA) diagnosis trajectory is debated, as several changes in diagnosis occur during PPA course, due to phenotype evolution from isolated language alterations to global cognitive impairment.

The goal of the present study, based on a French cohort was to describe the demographics and the evolution of subjects with (PPA) in comparison with Alzheimer Disease (AD) on a period of seven years.

Methods

We conducted a repeated cross-sectional study. The study population comprised individuals with PPA and AD diagnosis ( $N=167191)$ from 2010 to 2016 in the French National data Bank (BNA). Demographic variables, MMSE scores, diagnosis status at each visit and prescribed treatments were considered.

Results

From 2010 to 2016, 5186 patients were initially diagnosed with PPA, 162005 with AD. Compared to AD subjects, significant differences were found concerning: age (younger at first diagnosis for PPA), gender (more balanced in PPA), education level (higher in PPA) and MMSE score (higher in PPA).

Percentage of pending diagnosis, delay between first consultation and first diagnosis and the number of different diagnoses before the diagnosis of interest were significantly higher in PPA group compared to $A D$ group. Pharmacological and non-pharmacological treatments were significatively more recommended following PPA than AD diagnosis.

\section{Conclusion}

This study improves the knowledge of PPA epidemiology and has the potential to help adopting appropriate public health service policies. It supports the hypothesis that PPA is diagnosed later than AD. The PPA diagnosis increases the prescription of non-pharmacological treatments, especially speech and language therapy (SLT) that is the main treatment available and most effective when at the initial stage.

Trial registration

ClinicalTrials.gov identifier NCT03687112

\section{Background}

Current diagnosis classification identifies three PPA subtypes: The agrammatic subtype (nfavPPA), the semantic subtype (svPPA) and the logopenic subtype (IvPPA). 
The age of onset of PPA is usually between 50 and 65 years[1, 2]. PPA ultimately leads to dementia, and the survival duration is estimated between 10 and 15 years[3]. No disease-modifying pharmacological intervention treatment is available so far. However, non-pharmacological interventions, such as speech and language therapy (SLT), have proven to be useful to compensate and maintain functional communication.

Proper PPA diagnosis increases the opportunities of providing early appropriate clinical interventions, implementing coordinated care plans, managing symptoms, improving patient safety, cost savings, and postponing institutionalization[4]. The neurodegenerative diagnosis trajectory is still debated, as several changes in diagnosis occur during the course of PPA, due to phenotype evolution from isolated language alterations to global cognitive impairment with associated multiple neuropsychiatric symptoms $[5,6]$. Furthermore, IvPPA is considered as an atypical phenotype of Alzheimer's disease (AD), which further complicates diagnosis[7, 8]. Even if the PPA duration is estimated at about 6 years before dementia onset, language symptoms could represent the only set of signs for as many as 10-14 years.

After a few years of disease progression, deficits in other cognitive domains than language appear, such as episodic memory or executive functions. However, the language dysfunction remains the most salient feature throughout the degeneration process $[9,10]$. The diagnosis of PPA is a major challenge in clinical practice as this phenotype is complex and constantly evolving.

The main objective of this study was to describe the clinical characteristics and the evolution in diagnosis of PPA in comparison with AD, over a period of seven years, in a large cohort of memory-clinic patients. The secondary objectives were to determine if the diagnosis of PPA is more difficult to establish (more changes in diagnosis before the PPA diagnosis) and more delayed, compared to AD diagnosis, and to compare the two syndromes according to the recommended therapeutic approaches.

\section{Methods}

\section{Participants}

Participants of the present study were recruited from the French National data Bank (BNA) which is part of the French strategy to fight against dementia[11] and records information since the end of 2009. This database was created to provide information about the medical activity of the French memory centres in order to adapt healthcare provision, and generate epidemiologic knowledge on the diseases and the medical practices. The BNA includes a limited set of demographic, diagnostic and clinical, selected by a national consensus group. The number of collected variables was limited to facilitate and enhance care providers to participate to this national database. Data are collected from 536 memory units in France: 434 memory centres (secondary level), 28 resource and research memory centres (tertiary level) and 74 independent neurologists who expressed the willingness to participate.

Each time a person consults one of these centres, a clinical record is generated and transferred to the database. Therefore, one patient can figure more than once in the BNA, depending on the number of 
medical acts he/she underwent.

The following variables were considered in the present study: gender, age, living conditions, education (five levels according to the French education system, corresponding to the following categories: no formal education, primary school level [equivalent to $1-5$ years of education], secondary school level with 6-9 years of education, secondary school level with 10-12 years of education, and university level [over 12 years of education]), type of medical centre, referring modalities, score on the Mini Mental Score Examination (MMSE)[12] date of consultation, medical diagnosis and recommended treatments.

The BNA differentiates 38 diagnostic groups, based on International Classification of Diseases, Tenth Revision, ICD-10. For treatments, the BNA records the presence of a prescription at the time of the consultation for 6 groups of psychotropic drugs classified as follow, using ATC codes: antidepressant (N06A), anxiolytic (N05B), hypnotic (N05C), antipsychotic (N05A), cholinesterase Inhibitors (ChEls) (N06DA) and N-Methyl-D-aspartate receptor antagonist (NMDA antagonist) (N06DX01). No data is available on drug generics or brand names, nor on dosage. Psycho social intervention and rehabilitation are recorded too.

\section{Study design and participant selection}

Patients were selected in the BNA from January 1, 2010 to December 31, 2016. Patients with at least once a diagnosis of PPA or semantic dementia were included in the PPA group (including all PPA subtypes). Patients with at least once a diagnosis of $A D$, but never of PPA or semantic dementia were included in the $A D$ group.

Individuals who already had the diagnosis of interest when first registered in the database were included only if their first consultation for memory problems was in the same year or the year before the first visit. This was intended to exclude patients who had a diagnosis established for a long time, and to collect data at the time of the first diagnosis. To describe the whole population included in the study, we selected data at the first diagnosis of interest. Given the importance of cognitive status, only patients with at least one existing MMSE evaluation at less than 1 year before or after the first diagnosis of interest were considered in the analyses. (See Fig. 1)

[insert Fig. 1]

Figure 1: Flowchart: Selection of the participants included in the study.

\section{Statistical analysis}

Incident cases were defined as those first diagnosed during the study period. Incidence was calculated by dividing the total number of incident cases by the total number of person-years for the catchment area population over 7 years (data from the French national institute for statistical and economic studies INSEE). 
Descriptive analyses were conducted using percent and frequency for qualitative variables and mean with SD for quantitative variables. Variables associated with diagnosis (i.e., PPA, AD) were analysed using Student t-test for quantitative variables and chi-squared tests for qualitative variables.

The change in treatment and the change in psychosocial interventions were determined using the McNemar test. In all analyses, a p value less than 0.05 was considered significant.

Statistical analyses were done with SAS Enterprise Guide software, version 5.1 (SAS Institute, Cary, NC, USA).

\section{Results}

\section{Clinical characteristics of the $\mathbf{2}$ groups}

The PPA and the AD groups respectively included 5,186 and 162,005 patients. The incidence rate of PPA was 1.14 per 100,000 person-years, while the incidence rate of AD was 35.7 per 100,000 persons-years. Demographic characteristics of the two groups are presented in Table 1. Patients with PPA were significantly younger (mean $=73.7 ; S D=9.1$ years) than those with $A D$ (mean $=81.4 ; S D=8.0$ years $(p<$ $0.001)$, and this was observed in all age groups, except for the patients aged 80 years and older for which the opposite pattern was observed. As shown on Table 1, the sex ratio was more balanced in the PPA than in the AD group, and the educational level was higher in the PPA than in the AD group, with a larger proportion of patients with more than 6 years of education (secondary school level).

Compared to the AD group, the patients of the PPA group were more often referred by neurologists or other specialists and less by general practitioners. In PPA more often than in AD, the diagnosis was established in a tertiary centre, and patients lived farther from the centre.

In the PPA group, the MMSE score at first diagnosis was significantly higher than in $A D$, and patients were more to live in community compared to the patients with AD. 
Table 1

Demographic characteristics.

\begin{tabular}{|c|c|c|c|c|c|}
\hline & \multicolumn{2}{|c|}{ PPA $(n=5,186)$} & \multicolumn{2}{|c|}{$A D(n=162,005)$} & \multirow[b]{2}{*}{$p$-value } \\
\hline & Mean & [SD] & Mean & [SD] & \\
\hline Age when first diagnosed, years & 73.7 & [9.1] & 81.4 & [8.0] & $<.001$ \\
\hline \multirow[t]{2}{*}{ MMSE at \pm 1 year after diagnosis } & 19.5 & [7.3] & 17.9 & [5.9] & $<.001$ \\
\hline & $\mathrm{n}$ & $(\%)$ & $\mathrm{n}$ & $(\%)$ & $\mathrm{p}$-value \\
\hline Gender & & & & & $<.001$ \\
\hline Female & 2,887 & $(55.7)$ & 112,751 & $(69.6)$ & \\
\hline Male & 2,299 & $(44.3)$ & 49,254 & $(30.4)$ & \\
\hline Type of center & & & & & $<.001$ \\
\hline Memory clinic & 2,739 & $(52.8)$ & 120,750 & $(74.5)$ & \\
\hline Regional specialized memory clinic & 2,309 & $(44.5)$ & 37,361 & $(23.1)$ & \\
\hline Private neurologist & 138 & $(2.7)$ & 3,894 & $(2.4)$ & \\
\hline Age at first consultation following diagnosis, years & & & & & $<.001$ \\
\hline$<50$ & 38 & $(0.7)$ & 462 & $(0.3)$ & \\
\hline$[50 ; 55[$ & 89 & $(1.7)$ & 567 & $(0.4)$ & \\
\hline$[55 ; 60[$ & 205 & $(4.0)$ & 1,493 & $(0.9)$ & \\
\hline$[60 ; 65[$ & 533 & $(10.3)$ & 3,038 & $(1.9)$ & \\
\hline$[65 ; 70[$ & 807 & $(15.6)$ & 6,694 & $(4.1)$ & \\
\hline$[70 ; 75[$ & 966 & $(18.6)$ & 14,801 & $(9.1)$ & \\
\hline$[75 ; 80[$ & 1,191 & $(23.0)$ & 31,774 & $(19.6)$ & \\
\hline$[80 ; 85[$ & 923 & $(17.8)$ & 47,930 & $(29.6)$ & \\
\hline$\geq 85$ & 834 & (8.4) & 55,246 & $(34.1)$ & \\
\hline Education & & & & & $<.001$ \\
\hline No education & 205 & $(4.0)$ & 12,523 & (7.7) & \\
\hline Primary & 1,536 & $(29.6)$ & 75,615 & $(46.7)$ & \\
\hline Secondary first cycle & 1,067 & $(20.6)$ & 26,893 & $(16.6)$ & \\
\hline Secondary second cycle & 805 & $(15.5)$ & 14,715 & $(9.1)$ & \\
\hline
\end{tabular}




\begin{tabular}{|c|c|c|c|c|c|}
\hline \multirow[b]{2}{*}{ Superior } & \multicolumn{2}{|c|}{ PPA $(n=5,186)$} & \multicolumn{2}{|c|}{$A D(n=162,005)$} & \\
\hline & 1,022 & $(19.7)$ & 13,222 & $(8.2)$ & \\
\hline Unknown & 551 & $(10.6)$ & 19,037 & $(11.8)$ & \\
\hline Initially referred by & & & & & $<.001$ \\
\hline General practitioner & 2,839 & $(54.7)$ & 106,157 & $(65.5)$ & \\
\hline Neurologist & 1,152 & $(22.2)$ & 9,751 & $(6.0)$ & \\
\hline Other specialists & 592 & $(11.4)$ & 17,665 & $(10.9)$ & \\
\hline Direct & 233 & $(4.5)$ & 6,974 & (4.3) & \\
\hline Others & 370 & $(7.1)$ & 21,458 & $(13.3)$ & \\
\hline Community-dwelling & & & & & $<.001$ \\
\hline No & 333 & $(6.4)$ & 27,240 & $(16.8)$ & \\
\hline Yes & 4,853 & $(93.6)$ & 134,765 & $(83.2)$ & \\
\hline Patient location & & & & & $<.001$ \\
\hline Within $50 \mathrm{~km}$ from the memory clinic & 4,245 & $(81.9)$ & 148,844 & $(91.9)$ & \\
\hline Over $50 \mathrm{~km}$ from the memory clinic & 941 & $(18.2)$ & 13,161 & (8.1) & \\
\hline
\end{tabular}

Evolution in diagnosis.

As shown in Table 2, the delay between the first consultation for cognitive disorders (that could be prior the first record in the BNA) and the first diagnosis visit was significantly longer in the PPA than in the AD group.

The number of different diagnoses before the diagnosis of interest was significantly different in the PPA group than in the $A D$ group (see Table 2). The proportion of patients having received no other diagnosis before diagnosis of interest was lower in the PPA than in the AD group. However, the proportion of patients having received more than one diagnosis before diagnosis of interest was higher in the PPA than in the $A D$ group.

We analyzed the diagnoses made before the diagnosis of interest in each group (Table 3). Except "pending diagnosis", the most frequent diagnosis given before PPA diagnosis were AD (12.6\%) then subjective cognitive complaint, followed by non-amnestic mild cognitive impairment. Though before $A D$ diagnosis, except "pending diagnosis", it was most often amnestic mild cognitive impairment (14\%) then cognitive complaint then non-amnestic mild cognitive impairment.

The proportion of patients having received no other diagnosis after PPA was identified was lower than after an AD diagnosis was made (see Table 2). 
Table 2

Number of different diagnoses before and after first consultation.

\begin{tabular}{|c|c|c|c|c|c|}
\hline & \multicolumn{2}{|c|}{$\begin{array}{l}\operatorname{PPA}(n= \\
5,186)\end{array}$} & \multicolumn{2}{|c|}{$A D(n=162,005)$} & \multirow[b]{2}{*}{$\begin{array}{l}p- \\
\text { value }\end{array}$} \\
\hline & Mean & [SD] & Mean & [SD] & \\
\hline \multirow{2}{*}{$\begin{array}{l}\text { Period between first consultation and first diagnosis } \\
\text { (years) }\end{array}$} & 0.7 & [1.4] & 0.6 & [1.4] & $<.001$ \\
\hline & $n$ & (\%) & $\mathrm{n}$ & (\%) & $\begin{array}{l}p- \\
\text { value }\end{array}$ \\
\hline $\begin{array}{l}\text { Number of different diagnoses BEFORE first } \\
\text { consultation with diagnosis of interest }\end{array}$ & & & & & $<.001$ \\
\hline 0 & 2,892 & $(55.8)$ & 98,775 & (61.0) & \\
\hline 1 & 1,890 & $(36.4)$ & 54,709 & (33.8) & \\
\hline 2 & 338 & (6.5) & 7,536 & $(4.7)$ & \\
\hline 3 & 54 & $(1.0)$ & 880 & $(0.5)$ & \\
\hline 4 & 10 & $(0.2)$ & 95 & $(0.1)$ & \\
\hline 5 & 2 & $(0.0)$ & 10 & $(0.0)$ & \\
\hline $\begin{array}{l}\text { Number of different diagnoses AFTER first } \\
\text { consultation with the diagnosis of interest }\end{array}$ & & & & & $<.001$ \\
\hline 0 & 3,971 & (76.6) & 147,235 & $(90.9)$ & \\
\hline 1 & 992 & (19.1) & 13,234 & $(8.2)$ & \\
\hline 2 & 186 & (3.6) & 1,344 & $(0.8)$ & \\
\hline 3 & 33 & $(0.6)$ & 164 & $(0.1)$ & \\
\hline 4 & 2 & $(0.0)$ & 27 & $(0.0)$ & \\
\hline 5 & 2 & $(0.0)$ & 1 & $(0.0)$ & \\
\hline
\end{tabular}

However, the proportion of patients having received more than one diagnosis after the first diagnosis visit was higher in the PPA than in the AD group. Diagnoses registered after a PPA diagnosis, were most often PPA (72.7\%), then AD then behavioral variant of frontotemporal neurocognitive disorder then mixed neurocognitive disorder. And after $A D$ diagnosis, it was most frequent $A D$ diagnosis $(90.7 \%)$ then mixed neurocognitive disorder (Table 3). 
Table 3

Diagnoses before and after first diagnosis of interest.

\begin{tabular}{|c|c|c|c|c|}
\hline & \multicolumn{4}{|c|}{ Diagnosis BEFORE first diagnosis of interest } \\
\hline & \multicolumn{2}{|l|}{ PPA } & \multicolumn{2}{|l|}{$A D$} \\
\hline & $\mathrm{n}$ & (\%) & $\mathrm{n}$ & $(\%)$ \\
\hline Pending Diagnosis & 3,277 & $(49.6)$ & 83,967 & $(53.4)$ \\
\hline Alzheimer Disease (AD) & 835 & $(12.6)$ & 0 & $(0.0)$ \\
\hline Memory complaints & 492 & $(7.4)$ & 11,781 & $(7.5)$ \\
\hline $\mathrm{MCl}$ & 467 & $(7.1)$ & 10,256 & $(6.5)$ \\
\hline Amnestic $\mathrm{MCl}$ & 294 & $(4.4)$ & 21,974 & $(14.0)$ \\
\hline Huntington disease & 205 & $(3.1)$ & 754 & $(0.5)$ \\
\hline Mixed Dementia & 177 & $(2.7)$ & 11,348 & $(7.2)$ \\
\hline Anxiety disorder, depressive disorder & 177 & $(2.7)$ & 5,540 & $(3.5)$ \\
\hline Frontotemporal Lobar Degeneration (FTLD) & 90 & $(1.4)$ & 280 & $(0.2)$ \\
\hline \multirow[t]{4}{*}{ Others } & 596 & $(9.0)$ & 11,419 & $(7.3)$ \\
\hline & \multicolumn{4}{|c|}{ Diagnosis AFTER first diagnosis of interest } \\
\hline & \multicolumn{2}{|l|}{ PPA } & \multicolumn{2}{|l|}{$A D$} \\
\hline & $\mathrm{n}$ & $(\%)$ & $\mathrm{n}$ & $(\%)$ \\
\hline PPA & 10,760 & $(72.7)$ & 0 & $(0.0)$ \\
\hline Alzheimer Disease (AD) & 2,049 & $(13.8)$ & 318,769 & $(90.7)$ \\
\hline Frontotemporal Lobar Degeneration (FTLD) & 328 & $(2.2)$ & 672 & $(0.2)$ \\
\hline Pending Diagnosis & 323 & $(2.2)$ & 3,761 & $(1.1)$ \\
\hline Huntington disease & 229 & $(1.5)$ & 634 & $(0.2)$ \\
\hline Mixed Dementia & 223 & $(1.5)$ & 14,267 & $(4.1)$ \\
\hline $\mathrm{MCl}$ & 146 & $(1.0)$ & 1,924 & $(0.5)$ \\
\hline Amnestic $\mathrm{MCl}$ & 89 & $(0.6)$ & 2,546 & $(0.7)$ \\
\hline Others & 1,107 & $(7.5)$ & 27,576 & $(7.8)$ \\
\hline
\end{tabular}

\section{Evolution in treatment}


Patients with at least one BNA record before and after the diagnosis of interest and for whom the data about pharmacological $(N=1,622)$ and non-pharmacological treatment $(N=1,605)$ were registered were selected. The number of patients under pharmacological treatment was significantly higher after, than before the diagnosis of PPA, and this is true for the different psychotropic drugs and for anti-dementia treatments. More patients received non-pharmacological treatments after a PPA compared to before the diagnosis, with the most common intervention being speech-language therapy (See Table 4). The delay between diagnosis and starting speech-language therapy was $6.9(+/-6.3)$ months.

In the $A D$ group too, the number of patients under pharmacological treatment was significantly higher after, than before the diagnosis $(\mathrm{N}=42,571)$ (see Table 5), and this is also true for the different psychotropic drugs and for anti-dementia treatments. As for the PPA group, more patients received nonpharmacological treatments after receiving an AD diagnosis compared to before the diagnosis $(\mathrm{N}=$ 41,560). The delay between diagnosis and starting speech-language therapy was $9.0(+/-9.6)$ months.

\section{Discussion}

The present study, based on data gathered in the BNA, showed an incidence of PPA of 1.14/100,000 person-year, which is consistent with that was previously reported in FTLD and can be estimated in PPA 5 1213 .

Median age at first diagnosis was significatively lower in the PPA than in the AD group, which is in agreement with other studies that have shown that symptoms onset happened at a younger age in PPA than in AD[14]. However, the onset of PPA is known to occur before the age of 65[15] and the disease is described as a progressive language deficit occurring between 45 and 70 years old[16]. So, our results are not aligned to those of previous studied cohort and indicates possible bias of recruiting older people in Memory Centres, because they are mostly known as a diagnostic facility for memory and not language disorders.

The other possible explanation would be the proportion of different PPAs in the database and the lack of literature on the age of onset of an IvPPA.

Sex ratio was more balanced in the PPA than in the AD group, with $55.7 \%$ of females. Unless a majority of women was described as usual, sex ratio varies from one cohort to another in AD, in PPA group our results are in agreement with the literature[3].

The educational level was higher in the PPA than in the AD group, which is more than described in other studies[3] but in agreement with the fact of early onset dementia patients are more educated than patients with late onset dementia[17]. So, compared to other studies, we can suppose that PPA will be able to cope better with greater brain damage than $\mathrm{AD}[18]$.

The mean MMSE score at first diagnosis was higher in the PPA than in the AD group. But verbal items could be biased and nonverbal items also as understanding of verbal instruction and answering could be 
impaired[19].

About evolution of PPA diagnosis, the most frequently, the diagnosis stays PPA (72.7\%), including temporal variant of FTLD, then AD then behavioral variant of FTLD. These results are in agreement with literature values[3].

Regarding the initially diagnosed PPA patients, the natural evolution to a FTD in temporal or frontal variant diagnosis is concordant as the language impairment will be accompanied by a more global cognitive deficit. In cognitively impaired patients, the appearance of a global cognitive deficit or behavioural symptoms announces a future onset of dementia in the six following years of evolution, mostly with a frontal phenotype (75\%)[20].

The second evolution observed in our study is AD with $13,8 \%$. These results are in agreement with the fact of due to similar neuropathology and the clinical phenotype presented after the aphasic state, the logopenic subtype of PPA is considered as an atypical form of $A D[21]$.

Other evolutive profile are also reported in our study like Lewy body dementia (0.5\%), corticobasal degeneration (CBD) $(0.5 \%)$ and Progressive supranuclear palsy $(0.5 \%)$ in agreement with literature[22, 23$]$.

Indeed, some authors consider PPA-plus syndromes when aphasia is still not the only major deficit. They report that disinhibition by familiarity, blunted judgment, and difficulty in problem solving result in a personality change. Personality change and asymmetric extrapyramidal deficit occur frequently considering the PPA anatomical dysfunction evolution, which is supposed to be close to behavioural variant of FTD or CBD[24].

Also, initial language deficits are reported in one third of corticobasal dementia.

However, in our study some results are surprising, as the percentage of evolution in subjective complaint which is not a mode of evolution of PPAs and can brings into question on a possible diagnostic error at the beginning.

Our secondary objectives were to determine if diagnosis of PPA is more difficult to establish and more delayed than $A D$ and what are the related prescribed treatment over seven years of follow-up.

For patients diagnosed of PPA at the end of the follow-up, the first diagnosis was 12,7 \% Alzheimer's dementia, $7.1 \%$ subjective memory complaint, $4.4 \%$ non-amnestic mild cognitive impairment. Subjective memory complaint in the cohort is corresponding to the first consultation of the patient that could, at this time, stay at a subjective cognitive impairment report. We know that PPA patients can complain of memory loss and may perform poorly in standard tests of memory[25]. However, percentage of patients initially diagnosed with $A D$ or with a pending diagnosis $(49,6 \%)$ seem support the hypothesis that diagnosis of PPA is more difficult and more delayed than AD.

Other results seem to support this hypothesis, like: 
- -the delay between the first consultation for cognitive troubles and the first diagnosis that is significantly longer than in AD group $(p<0.001)$.

- -the number of different diagnoses before the diagnosis of interest that is significantly higher in PPA group (0.5+/- 0.7 (mean $\pm S D)$ ) than in AD group $(0.4+/-0.6)$.

- the percentage of patients with more than one diagnosis that was higher in PPA group than in AD group.

To summarize, despite the evolution of the diagnostic criteria of Primary Progressive Aphasia over time[16, 26], their diagnostic still does not seem optimal.

Our study shows also that PPA diagnosis is particularly important because modifies pharmacological and nonpharmacological interventions.

Indeed, the number of patients under pharmacological treatment increases of more than $50 \%$ after the diagnosis of PPA. Also, non-pharmacological treatments increase after the diagnosis and especially speech therapy $(22.7 \%$ before and $66.0 \%$ after).

In the field of pharmacological interventions, to date, no medications have been shown to improve or stabilize cognitive deficits in patients with PPA[27]. Indeed, clinical trials on patients with FTD and controlled trials on PPA patients with bromocriptine[28] and memantine[29] have not demonstrated any efficiency. Studies on limited patient samples with, galantamine, rivastigmine, and selegiline have shown no results[30]. Also, although some patients with PPA, particularly with a logopenic subtype, may suffer from atypical $A D$, cholinesterase inhibitors have shown no results. No effect is expected as cholinergic deficit has been specifically identified in AD's physiopathology. Worsening of behavioural variant of FTD is also suspected under anticholinerterasic treatment. So on, actual recommendation is not favourable for anticholinesterase inhibitors use in FTD[13].

However, benefits have been found on behaviour but not on cognition with the cholinesterase inhibitor rivastigmine and the MAO-B inhibitor selegiline[31].

The use of antidepressants, such as trazadone, is known to be effective on the behavioural symptoms but has no impact on cognition is reported in FTD[32]. Antipsychotics with careful and limited use could improve behavioural symptoms but their side effect on wake and cognition limits their use. It's suspected that high-frequency repetitive transcranial magnetic stimulation (hf-rTMS) applied to the left prefrontal cortex produces improvement on language test that seems to last, other magnetic stimulations are suspected to maintain some language capacities[33].

In summary, although no drugs have shown an effectiveness on PPA. But, on behavioural disorders, some drugs have been deleterious and others had positive effects. It thus appears essential to establish a diagnosis of PPA to set up an adapted medical treatment. 
A key intervention on the PPA population is speech and language therapy (SLT): a specific form of cognitive intervention that evaluates communication skills and designs a personalized intervention plan to improve communication abilities. This type of intervention has been shown to be effective and advised to be implemented in a more systematic way[34].

Also, in addition to the take care of language disorders, speech therapy is particularly important for swallowing disorders, which represent vital risks. Indeed, all variants showed swallowing difficulties and they were more frequent in PPA-S[35].

Finally, the put of diagnosis is essential whether it is for more adapted pharmacological or nonpharmacological interventions.

\section{Limitations of the present study}

Despite BNA represents a valuable epidemiologic tool because it grants access to many patients with dementia and permits follow-up studies, several limitations should be noted.

First, data are entered into the BNA by different physicians and even though they all follow standard criteria for diagnosis, there is no external validation that those criteria were met. Also, criteria for diagnosis of PPA have be modified since 2010 (semantic dementia). Second, even though the BNA includes the great majority of individuals with PPA and associated disorders who are referred to specialized centres (French memory units), individuals included in the BNA are not fully representative of the total French population with PPA. Indeed, one part of the population with PPA is under general practitioner (GP) supervision only (GPs do not currently have access to the BNA), and another part of the population is referred to specialists (geriatricians, neurologists, psychiatrists) who are not using the BNA database.

\section{Conclusion}

This study provided data on the clinical characteristics and the evolution of PPAs over a very large cohort. It has also highlighted:

1.the current difficulty of making a diagnosis of PPA because of the varied symptomatology of the three variants and the underlying pathology (FTD or AD).

2. the use of poorly adapted diagnostic and severity assessment tools due to verbal instructions and proposed language tasks.

3. the importance of early diagnosis between PPA and AD due to differences in therapeutic approaches.

The perspectives of this study are to develop tools of diagnosis and severity assessment more adapted by including early markers of the pathology (graphic markers and vocal markers). 


\section{Declarations}

\section{Ethics approval and consent to participate}

The study was declared to the National Commission for Information Technology and Civil Liberties (CNIL) the number R04-051. All clinical work was conducted in compliance with current Good Clinical Practices as referenced in the International Conference on Harmonisation of Technical Requirements for Registration of Pharmaceuticals for Human Use guidelines, local regulatory requirements, and the principles of the Declaration of Helsinki. All patients enrolled in the study provided information before their participation. All clinical sites were required to sign a study-specific site blinding plan. This study is registered on ClinicalTrials. gov (NCT03687112).

\section{Consent for publication}

Not applicable

\section{Availability of data and materials}

The data reported are part of an ongoing registration program. Deidentified participant data are not available for legal and ethical reasons. Anonymised data will be made available for research purposes, upon request and specifical approval of the database advisory board and ethical committee.

\section{Competing interests}

The authors declare that they have no competing interests

\section{Funding}

The French National Alzheimer Database (Banque Nationale Alzheimer) is funded by the French Ministry of Health.

\section{Authors' contributions}

$A M, A P, V M$ and $A G$ contributed to the study concept and design, were involved in data synthesis and data interpretation, and drafted the work. RF performed the statistical analysis. JM and MT contributed to draft the work. PHR and AM was involved in the supervision and quality control of the BNA database. All authors revised the final manuscript and approved it.

\section{Acknowledgements}

Not applicable

\section{References}


1. Ratnavalli E, Brayne C, Dawson K, Hodges JR. The prevalence of frontotemporal dementia. Neurology. 2002 Jun 11;58(11):1615-21.

2. Mesulam M-M, Rogalski EJ, Wieneke C, Hurley RS, Geula C, Bigio EH, et al. Primary progressive aphasia and the evolving neurology of the language network. Nature Reviews Neurology. 2014 Oct;10(10):554-69.

3. Le Rhun E, Richard F, Pasquier F. Natural history of primary progressive aphasia. Neurology. 2005 Sep 27;65(6):887-91.

4. Dubois B, Padovani A, Scheltens P, Rossi A, Dell'Agnello G. Timely Diagnosis for Alzheimer's Disease: A Literature Review on Benefits and Challenges. Saykin A, editor. Journal of Alzheimer's Disease. 2015 Oct 17;49(3):617-31.

5. Kertesz A, Morlog D, Light M, Blair M, Davidson W, Jesso S, et al. Galantamine in Frontotemporal Dementia and Primary Progressive Aphasia. Dementia and Geriatric Cognitive Disorders. 2008;25(2):178-85.

6. Grossman M. Primary progressive aphasia: clinicopathological correlations. Nature Reviews Neurology. 2010 Feb;6(2):88-97.

7. Dubois B, Feldman HH, Jacova C, Cummings JL, Dekosky ST, Barberger-Gateau P, et al. Revising the definition of Alzheimer's disease: a new lexicon. Lancet Neurol. 2010 Nov;9(11):1118-27.

8. Spinelli EG, Mandelli ML, Miller ZA, Santos-Santos MA, Wilson SM, Agosta F, et al. Typical and atypical pathology in primary progressive aphasia variants: Pathology in PPA Variants. Ann Neurol. 2017 Mar;81(3):430-43.

9. Mesulam M. Primary progressive aphasia: A dementia of the language network. Dement Neuropsychol. 2013 Jan 1;7(1):2-9.

10. Weintraub S, Rubin NP, Mesulam MM. Primary progressive aphasia. Longitudinal course, neuropsychological profile, and language features. Arch Neurol. 1990 Dec;47(12):1329-35.

11. Spinney L. Alzheimer's disease funding and the French health system. The Lancet Neurology. 2008 Jan;7(1):26-7.

12. Folstein MF, Folstein SE, McHugh PR. "Mini-mental state". A practical method for grading the cognitive state of patients for the clinician. J Psychiatr Res. 1975 Nov;12(3):189-98.

13. Kerchner GA, Tartaglia MC, Boxer AL. Abhorring the vacuum: use of Alzheimer's disease medications in frontotemporal dementia. Expert Review of Neurotherapeutics. 2011 May;11(5):709-17.

14. Hommet C, Mondon K, Perrier D, Rimbaux S, Autret A, Constans T. L'aphasie progressive primaire : un cadre à part dans les pathologies neurodégénératives. La Revue de Médecine Interne. 2008 May 1;29(5):401-5.

15. Mesulam M-M, Wieneke C, Thompson C, Rogalski E, Weintraub S. Quantitative classification of primary progressive aphasia at early and mild impairment stages. Brain. 2012 May 1;135(5):153753.

16. Mesulam MM. Primary progressive aphasia. Ann Neurol. 2001 Apr;49(4):425-32. 
17. Maiovis $P$, loannidis $P$, Konstantinopoulou E, Karacostas D. Early onset degenerative dementias: demographic characteristics and etiologic classification in a tertiary referral center. Acta Neurologica Belgica. 2015 Mar;115(1):27-31.

18. Maiovis P, Ioannidis P, Gerasimou G, Gotzamani- Psarrakou A, Karacostas D. Cognitive Reserve Hypothesis in Frontotemporal Dementia: Evidence from a Brain SPECT Study in a Series of Greek Frontotemporal Dementia Patients. Neurodegenerative Diseases. 2018;18(2-3):69-73.

19. Croisile B, Astier J-L, Beaumont C. Diagnostic d'une aphasie progressive primaire non-fluente en pratique clinique courante. /data/revues/00353787/01590011/1009/ [Internet]. 2008 Mar 1 [cited 2019 May 5]; Available from: https://www.em-consulte.com/en/article/104476

20. Signoret J-L, Allard M, Benoit N, Bolgert F. Evaluation des troubles de mémoire et des désordres cognitifs associés: B.E.C. 96. Paris, France: IPSEN; 1989. 60 p.

21. McKhann GM, Knopman DS, Chertkow H, Hyman BT, Jack CR, Kawas CH, et al. The diagnosis of dementia due to Alzheimer's disease: Recommendations from the National Institute on AgingAlzheimer's Association workgroups on diagnostic guidelines for Alzheimer's disease. Alzheimer's \& Dementia. 2011 May;7(3):263-9.

22. Kertesz A, Davidson W, Mccabe P, Takagi K, Munoz D. Primary progressive aphasia: Diagnosis, varieties, evolution. Journal of the International Neuropsychological Society. 2003 Jul;9(5):710-9.

23. Knibb JA, Xuereb JH, Patterson K, Hodges JR. Clinical and pathological characterization of progressive aphasia. Annals of Neurology. 2006 Jan;59(1):156-65.

24. Mesulam M-M. Primary Progressive Aphasia - A Language-Based Dementia. New England Journal of Medicine. 2003 Oct 16;349(16):1535-42.

25. Weintraub S, Rogalski E, Shaw E, Sawlani S, Rademaker A, Wieneke C, et al. Verbal and Nonverbal Memory in Primary Progressive Aphasia: The Three Words-Three Shapes Test. Behavioural Neurology. 2013;26(1-2):67-76.

26. Gorno-Tempini ML, Hillis AE, Weintraub S, Kertesz A, Mendez M, Cappa SF, et al. Classification of primary progressive aphasia and its variants. Neurology. 2011 Mar 15;76(11):1006-14.

27. Shigaeff N, Zanetti M, Tierno S de A, Tommaso ABGD, Marques TC, Franco FG de M. An interdisciplinary approach aiding the diagnosis of primary progressive aphasia: A case report. Dementia \& Neuropsychologia. 2017 Mar;11(1):100-2.

28. Reed DA, Johnson NA, Thompson C, Weintraub S, Mesulam M-M. A clinical trial of bromocriptine for treatment of primary progressive aphasia. Annals of Neurology. 2004 Nov;56(5):750-750.

29. Johnson NA, Rademaker A, Weintraub S, Gitelman D, Wienecke C, Mesulam M. Pilot trial of memantine in primary progressive aphasia. Alzheimer Dis Assoc Disord. 2010 Sep;24(3):308.

30. Birks J, Flicker L. Selegiline for Alzheimer's disease. Cochrane Database Syst Rev. 2003; (1):CD000442.

31. Moretti R, Torre P, Antonello RM, Cattaruzza T, Cazzato G, Bava A. Rivastigmine in frontotemporal dementia: an open-label study. Drugs Aging. 2004;21(14):931-7. 
32. Lebert F, Stekke W, Hasenbroekx C, Pasquier F. Frontotemporal dementia: a randomised, controlled trial with trazodone. Dement Geriatr Cogn Disord. 2004;17(4):355-9.

33. Köhler TS, Choy JT, Fazili AA, Koenig JF, Brannigan RE. A critical analysis of the reported association between vasectomy and frontotemporal dementia. Asian Journal of Andrology. 2012 Nov;14(6):9034.

34. Kiousi V, Arnaoutoglou M, Printza A. Speech and language intervention for language impairment in patients in the FTD-ALS spectrum. Hell J Nucl Med. 2019 Apr;22 Suppl:133-46.

35. Marin S de MC, Bertolucci PHF, Marin LF, de Oliveira FF, Wajman JR, Bahia VS, et al. Swallowing in primary progressive aphasia. NeuroRehabilitation. 2016 Feb 10;38(1):85-92.

\section{Figures}




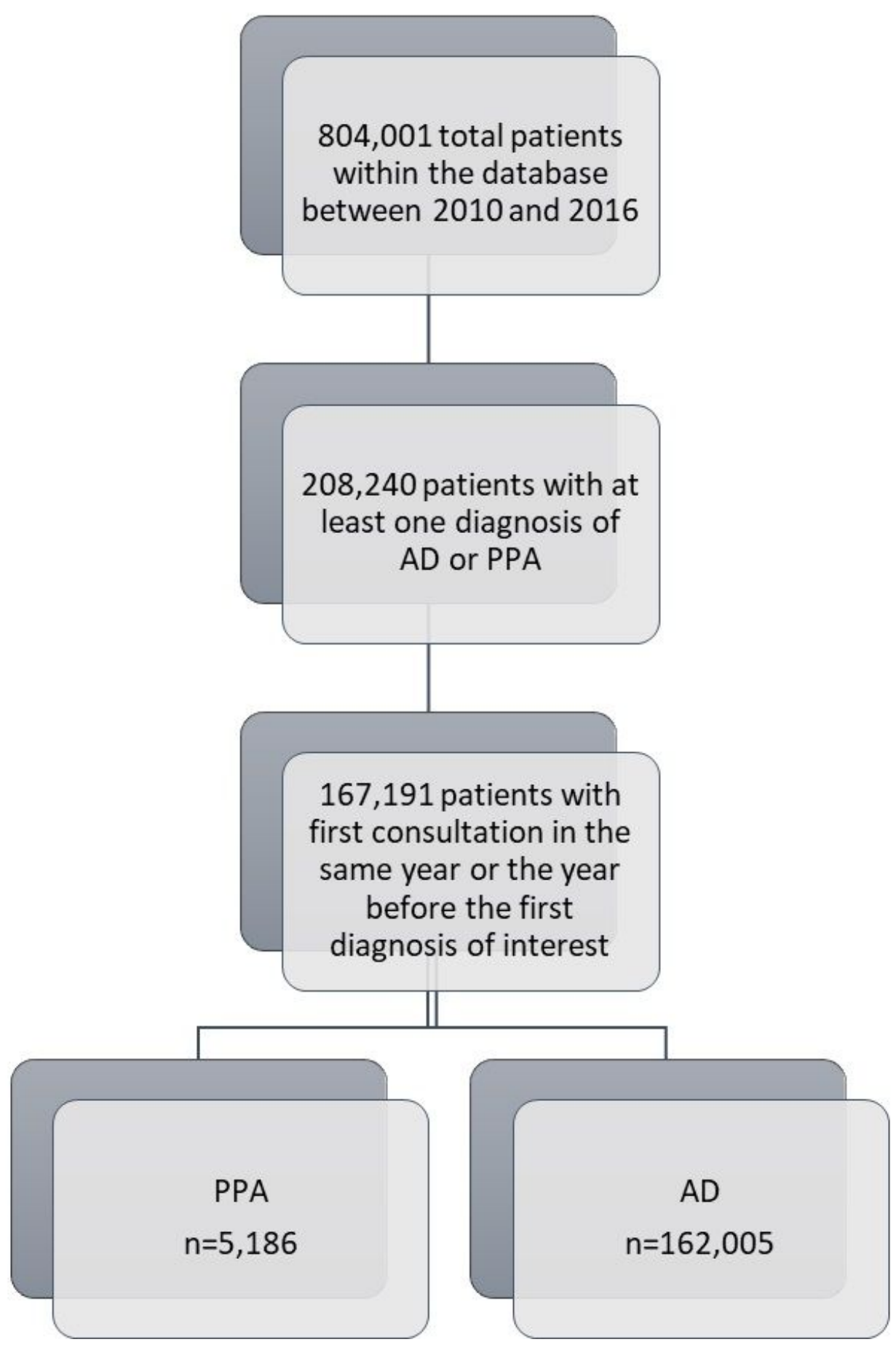

Figure 1

Flowchart: Selection of the participants included in the study. 